\title{
On the Polarizability of a Cluster of Interacting Particles in Polarization Radiation
}

\author{
D. Yu. Sergeeva ${ }^{a}$ and A. A. Tishchenko ${ }^{a, b, c, *}$ \\ ${ }^{a}$ National Research Nuclear University MEPhI (Moscow Engineering Physics Institute), Moscow, 115409 Russia \\ ${ }^{b}$ Laboratory of Radiation Physics, Belgorod National Research University, Belgorod, 308015 Russia \\ ${ }^{c}$ National Research Centre “Kurchatov Institute”, Moscow, 123182 Russia \\ *e-mail: tishchenko@mephi.ru \\ Received April 29, 2020; revised May 5, 2020; accepted May 5, 2020
}

\begin{abstract}
The study considers diffraction radiation, which is excited when an electron moves near a cluster of two interacting subwavelength particles. The interaction is manifested in the fact that the radiation field from each particle is determined not only by the external field of the electron, but also by the field of the neighboring particle. Based on the obtained expressions for the radiation field, the function of cluster polarizability is determined. It characterizes the cluster response to the field of the electron as a whole. It is interesting that the obtained response function of the cluster to an external field, even in the framework of linear theory, generally depends on the external field itself.
\end{abstract}

Keywords: polarizability, polarization radiation, interaction

DOI: $10.1134 / \mathrm{S} 1063778820090239$

\section{INTRODUCTION}

Polarization radiation is any radiation from charged particles that is excited not by changing the modulus or direction of velocity, as in the case of bremsstrahlung or synchrotron radiation, but by inducing the dynamic polarization of matter by the particle's own field. In other words, the direct source of radiation is not a charged particle, but a substance. The polarization type of radiation involves transition radiation, diffraction radiation, Vavilov-Cherenkov radiation, Smith-Purcell radiation (SPR), which is a special case of diffraction radiation from a periodic target, and parametric $\mathrm{X}$-ray radiation.

In general, this study is devoted to polarization radiation, and the calculation was carried out using diffraction radiation (DR) as an example [1]. For brevity, below we will talk only about DR, bearing in mind that everything that was said refers to polarization radiation in general everywhere, where otherwise it is not specified separately. A necessary condition for the excitation of this type of radiation is the presence of target inhomogeneity along the particle trajectory. The key feature is that the trajectory of charged particles passes outside the target, and the radiation process itself is not a direct consequence of scattering of charges on the target material. When exciting DR, the properties of the beam of charged particles practically do not change, which opens the possibility of using DR for non-disturbing diagnostics of bunches [2-4].
DR also is the basis of an efficient, powerful source of electromagnetic radiation [5], including SPR-based free electron lasers [6]. SPR, like DR, finds application in diagnostics of charged particle bunches [7]. This is due to such characteristic properties of the SPR as monochromaticity and large characteristic angles of observation relative to the particle trajectory.

An active investigation of DR from ordered arrays, whose elements are much smaller than the wavelength of radiation, started with the development of nanoelectronics and nanotechnology [8-11]. In the literature, such one-dimensional, two-dimensional, or three-dimensional periodic targets are often called photonic and plasmonic crystals, metasurfaces. Exploring these problems is mainly related to new possibilities for diagnostics of substances and medical applications.

In recent years, a series of studies appeared in which the authors investigated SPR from singleperiod gratings with simultaneous excitation of surface plasmons, which significantly enhance the radiation $[12,13]$. In [14], on an example of a cluster of two particles, it was shown that radiation enhancement is possible not only due to the excitation of plasmons on the surface of such structures, but also due to the interaction between elements. The interaction effect manifests itself in the shift in radiation frequencies, splitting of maxima, and the appearance of new intensity maxima. It is noteworthy that the interaction effect takes place not only at close distances between particles, but 


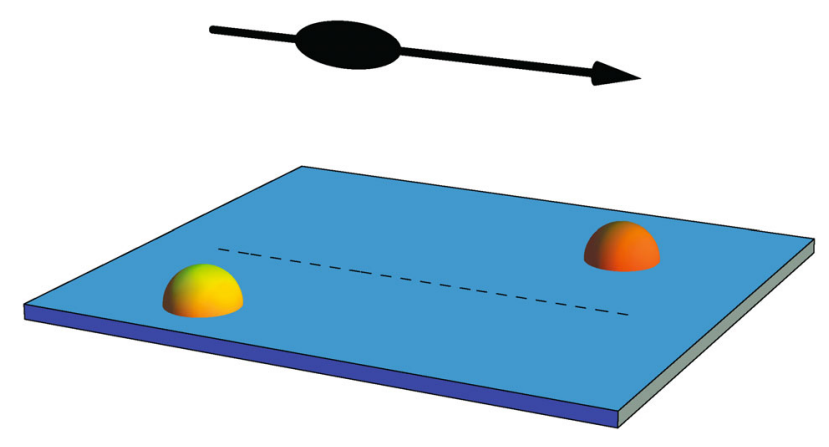

Fig. 1. Scheme of the DR excitation by an electron flying past a cluster of two particles.

also their "long-range" interaction is observed. This means that the interaction should be considered not only between nearest elements, as is traditionally done in the transition to a macroscopic description, but between all elements. For chaotically located and infinitely large arrays of particles, averaging can be performed over the position of all particles in the cluster with a certain weight function. This is difficult for a limited number of strictly ordered particles in the cluster. The radiation field calculation with allowance for all interactions between particles is reduced to solving a large number of self-consistent equations, which is difficult for clusters with the number of particles starting, for example, from 10.

In this paper, with an example of two particles, we will show how the interaction between particles in a cluster can be considered by introducing effective polarizability of particles. The essence of introducing effective polarizability is to consider the field from neighbouring particles in the response function of one particle.

\section{DIFFRACTION RADIATION \\ FROM A CLUSTER OF TWO PARTICLES}

Let us consider the excitation of DR during the flight of an electron with charge $e$ and constant velocity $\mathbf{v}$ near a cluster of two particles (Fig. 1). The particles are located in one plane, the electron moves at constant distance $h$ from this plane. We choose the coordinate system in such a way that the origin of coordinates lies on the plane on which the particles are located, the $O x$ axis coincides with the direction of the electron velocity, the $O z$ axis coincides with the normal to the plane of particles location, and the electron moves in the positive half-space, $z>0$.

Let the microparticles have different sizes $r_{\alpha}$ and $r_{\beta}$ and different polarizabilities $\alpha(\omega)$ and $\beta(\omega)$. The condition that the particle size is small in comparison with the wavelength of the emitted waves, $r_{\alpha}, r_{\beta} \ll \lambda$ makes it possible to use the dipole approximation to calculate the radiation field from two interacting particles. Moreover, let the position of the particles be deter- mined by radius-vectors $\mathbf{R}_{a}$ and $\mathbf{R}_{b}$. The radiation field can be calculated by solving the Maxwell system of equations, taking into account the fact that the current density is

$$
\mathbf{j}(\mathbf{r}, t)=\mathbf{j}_{0}(\mathbf{r}, t)+\mathbf{j}_{a}(\mathbf{r}, t)+\mathbf{j}_{b}(\mathbf{r}, t)
$$

where $\mathbf{j}_{0}$ describes the density current of a charged particle:

$$
\mathbf{j}_{0}(\mathbf{r}, t)=e \mathbf{v} \delta\left(\mathbf{r}-\mathbf{v} t-h \mathbf{e}_{z}\right),
$$

where $\delta$ is the Dirac delta-function, $\mathbf{j}_{a}$ and $\mathbf{j}_{b}$ are the densities of polarization currents appearing in each particle. The Fourier image of these currents is

$$
\mathbf{j}_{a}(\mathbf{r}, \omega)=-i \omega \alpha(\omega) \mathbf{E}^{\mathrm{act}}\left(\mathbf{R}_{a}, \omega\right) \delta\left(\mathbf{r}-\mathbf{R}_{a}\right),
$$

where $\mathbf{E}^{\text {act }}$ is the total field acting on a microparticle. Following the approach outlined in [14], we obtain the $i$-th component of the field at long distances $k r \gg 1$ in form

$$
\begin{gathered}
E_{i}^{\mathrm{rad}}(\mathbf{r}, \omega)=\frac{e^{i k r}}{r}\left(k^{2} \delta_{i s}-k_{i} k_{s}\right)\left\{\alpha(\omega) e^{-i \mathbf{k} \mathbf{R}_{a}}\right. \\
\times\left(E_{s}^{0}\left(\mathbf{R}_{a}, \omega\right)+E_{1 s}\left(\mathbf{R}_{a}, \omega\right)\right) \\
\left.+\beta(\omega) e^{-i \mathbf{k} \mathbf{R}_{b}}\left(E_{s}^{0}\left(\mathbf{R}_{b}, \omega\right)+E_{2 s}\left(\mathbf{R}_{b}, \omega\right)\right)\right\},
\end{gathered}
$$

where $\delta_{i s}$ is the Kronecker symbol, $\mathbf{k}$ is the wave-vector, $E_{s}^{0}\left(\mathbf{R}_{a}, \omega\right)$ is the Fourier image of the $s$-component of the field of the electron at point $\mathbf{R}_{a}$ :

$$
\begin{gathered}
\mathbf{E}^{0}(\mathbf{r}, \omega)=-\frac{i e}{\pi V} e^{i \frac{\underline{\omega}_{x}}{V}} \frac{\omega}{c \beta \gamma} \\
\times\left[\frac{1}{\gamma} K_{0}\left(\frac{\omega}{c \beta \gamma} \rho\right) \mathbf{e}_{x}+i \frac{\mathbf{\rho}}{\rho} K_{1}\left(\frac{\omega}{c \beta \gamma} \rho\right)\right] \\
\mathbf{\rho}=y \mathbf{e}_{y}+(z-h) \mathbf{e}_{z}, \text { and } \\
E_{1 s}\left(\mathbf{R}_{a}, \omega\right)=-e^{i R k} \frac{\beta(\omega)}{V} t_{s j}^{(1)} E_{j}^{0}\left(\mathbf{R}_{b}, \omega\right) \\
+\beta(\omega) e^{2 i R k} \frac{\alpha(\omega)}{V} t_{s j}^{(2)} E_{j}^{0}\left(\mathbf{R}_{a}, \omega\right), \\
E_{2 s}\left(\mathbf{R}_{b}, \omega\right)=-e^{i R k} \frac{\alpha(\omega)}{V} t_{s j}^{(1)} E_{j}^{0}\left(\mathbf{R}_{a}, \omega\right) \\
+\beta(\omega) e^{2 i R k} \frac{\alpha(\omega)}{V} t_{s j}^{(2)} E_{j}^{0}\left(\mathbf{R}_{b}, \omega\right), \\
t_{s j}^{(1)}=B \delta_{s j}-\frac{\alpha(\omega) \beta(\omega) e^{2 i R k}}{A B(A+B)+A} \frac{R_{s} R_{j}}{R^{2}}, \\
t_{s j}^{(2)}=B^{2} \delta_{s j}-\frac{A(A+2 B)}{W} \frac{R_{s} R_{j}}{R^{2}}
\end{gathered}
$$

and we used the notations: 


$$
\begin{gathered}
V=1-\alpha(\omega) \beta(\omega) e^{2 i R k} B^{2}, \\
W=\alpha(\omega) \beta(\omega) e^{2 i R k}(A+B)^{2}-1, \\
A=\frac{k^{2} R^{2}+3 i k R-3}{R^{3}}, \\
B=-\frac{k^{2} R^{2}+i k R-1}{R^{3}}, \quad R=\left|\mathbf{R}_{a}-\mathbf{R}_{b}\right| .
\end{gathered}
$$

The resonance conditions follow from expression (4), i.e., the conditions for a sharp radiation enhancement due to local field effects caused by the interaction of particles:

$$
\begin{gathered}
1=\operatorname{Re}\left[\alpha^{2}(\omega) e^{2 i R k} B^{2}\right] . \\
1=\operatorname{Re}\left[\alpha^{2}(\omega) e^{2 i R k}(A+B)^{2}\right] .
\end{gathered}
$$

Similarly to this, solving the problem of radiation from a single particle with polarizability $\alpha_{\text {eff }}(\omega)$, located at point $\mathbf{R}_{c}=\left(\mathbf{R}_{a}+\mathbf{R}_{b}\right) / 2$, we obtain the expression for the $i$-th component of the radiation field at large distances

$$
\begin{aligned}
E_{i}^{\mathrm{rad}}(\mathbf{r}, \omega) & =\frac{e^{i k r}}{r}\left(k^{2} \delta_{i s}-k_{i} k_{s}\right) \alpha_{\mathrm{eff}}(\omega) \\
& \times e^{-i \mathbf{k} \mathbf{R}_{c}} E_{s}^{0}\left(\mathbf{R}_{c}, \omega\right) .
\end{aligned}
$$

Let us compare expression (4) with similar expression (9) for radiation from one particle with effective polarizability $\alpha_{\text {eff }}(\omega)$. From the comparison, we obtain an expression for $\alpha_{\text {eff }}(\omega)$ :

$$
\begin{gathered}
\alpha_{\mathrm{eff}}(\omega)=\mid\left[\mathbf{k},\left.\left[\mathbf{k}, e^{-i \mathbf{R}_{c} \mathbf{k}} \mathbf{E}^{0}\left(\mathbf{R}_{c}, \omega\right)\right]\right|^{-1}\right. \\
\times\left[\mathbf{k},\left[\mathbf{k}, \alpha(\omega) e^{-i \mathbf{k} \mathbf{R}_{a}} \mathbf{E}^{0}\left(\mathbf{R}_{a}, \omega\right)+\beta(\omega) e^{-i \mathbf{k} \mathbf{R}_{b}} \mathbf{E}^{0}\left(\mathbf{R}_{b}, \omega\right)\right]\right] \\
+\left[\mathbf{k},\left[\mathbf{k}, \beta(\omega) e^{-i \mathbf{k} \mathbf{R}_{b}} \mathbf{E}_{2}\left(\mathbf{R}_{b}, \omega\right)+\alpha(\omega) e^{-i \mathbf{k} \mathbf{R}_{a}} \mathbf{E}_{1}\left(\mathbf{R}_{a}, \omega\right)\right]\right] \mid .
\end{gathered}
$$

Thus, the radiation field from a cluster of two interacting particles is determined by expressions (6), (9), and (10).

Polarizability $\alpha_{\text {eff }}(\omega)$ characterizes the response of a pair of microparticles as a whole to an external electric field. As we can see, it is remarkable that the effective polarizability of a cluster of two particles depends not only on the polarizability of particles forming it and on their relative position, including the distance between them, but also on the external field. This fact is important since in linear theory the response function of the system is usually assumed to be independent of the field of external sources.

Note also that the approach implemented here enables one to determine only the real part of the polarizability function, which, generally speaking, has also, an imaginary component.

\section{EFFECTIVE POLARIZABILITY IN A PLANE WAVE FIELD}

Note that all the formulas obtained above are written in terms of an external field, namely the field of electron $E_{s}^{0}\left(\mathbf{R}_{a}, \omega\right)$. However, when obtaining the results, we did not use the explicit form of the field of external sources, therefore, generally speaking, the external field can be arbitrary. In order to verify whether the obtained formulas really give a correct transition to the previously known cases, we consider a cluster of two noninteracting particles in the external field of a plane wave. Then, expression (4) remains valid, but now $E_{s}^{0}\left(\mathbf{R}_{a}, \omega\right)$ is the Fourier image of plane wave field

$$
\mathbf{E}^{0}\left(\mathbf{R}_{a}, \omega\right)=\mathbf{E}^{0} e^{i \mathbf{k}_{0} \mathbf{R}_{a}} \delta\left(\omega-\omega_{0}\right),
$$

and fields $\mathbf{E}_{2}\left(\mathbf{R}_{b}, \omega\right)$ and $\mathbf{E}_{1}\left(\mathbf{R}_{a}, \omega\right)$ are proportional to (11). In contrast to the field of the electron, which has a more complex dependence on the particle coordinates, the coordinate enters only as a phase factor in the field of a plane wave. It is easy to show in this case that expression (10) for the effective polarizability takes form

$$
\alpha_{\text {eff }}(\omega)=\alpha(\omega) e^{i\left(\mathbf{k}-\mathbf{k}_{0}\right)\left(\mathbf{R}_{b}-\mathbf{R}_{a}\right) / 2}+\beta(\omega) e^{-i\left(\mathbf{k}-\mathbf{k}_{0}\right)\left(\mathbf{R}_{b}-\mathbf{R}_{a}\right) / 2} .
$$

Thus, as expected, the effective polarizability of a system of two noninteracting particles depends only on the properties of particles and the distance between them, determined by vector $\mathbf{R}_{b}-\mathbf{R}_{a}$, but not on the external field. It is easy to see that in the case of particle $\beta(\omega)=0$ and $\alpha_{\text {eff }}(\omega)$ coincides with $\alpha(\omega)$ up to a phase factor, the presence of which is simply due to the choice of the cluster center at point $\mathbf{R}_{c}=\left(\mathbf{R}_{a}+\mathbf{R}_{b}\right) / 2$.

\section{CONCLUSIONS}

In this study, we considered diffraction radiation, which is excited during the flight of an electron near a cluster of two interacting subwavelength particles. In the case of two particles, the interaction can be taken into account completely analytically by solving two self-consistent tensor equations. As shown above, such a cluster of two particles, or a dimer, can be represented as a single radiation source with certain effective polarizability $\alpha_{\text {eff }}(\omega)$. If the particles interact, i.e., the radiation field from each is determined not only by the external field of the electron, but also by the fields from neighboring particles, then $\alpha_{\text {eff }}(\omega)$ is determined by formula (10). The problem was solved within the limits of applicability of the dipole approximation, that is, the size of the cluster is small in comparison with the wavelength of radiation. Despite the apparent severity of this limitation, of interest, is the problem of excitation of terahertz radiation from micro- and nanoclusters. The obtained conditions for the enhancement of radiation due to interaction open the possibility of practical use of the results in generation 
of intense radiation, as well as in the field of diagnostics of relativistic electron beams, nano- and microstructures.

\section{FUNDING}

The study was supported by the Russian Science Foundation (project no. 19-72-00178).

\section{CONFLICT OF INTEREST}

The authors declare that they have no conflicts of interest.

\section{REFERENCES}

1. S. J. Smith and E. M. Purcell, Phys. Rev. 92, 1069 (1953).

2. A. P. Potylitsyn, M. I. Ryazanov, M. N. Strikhanov, and A. A. Tishchenko, Diffraction Radiation from Relativistic Particles (Springer, Berlin, 2010).
3. P. Karataev et al., Phys. Rev. Lett. 93, 244802 (2004).

4. A. Cianchi et al., New J. Phys. 16, 113029 (2014).

5. I. V. Konoplev et al., Phys. Rev. A 84, 013826 (2011).

6. D. Li et al., Appl. Phys. Lett. 100, 191101 (2012).

7. H. L. Andrews et al., Phys. Rev. Spec. Top. Accel. Beams 17, 052802 (2014).

8. F. J. Garcia de Abajo, Phys. Rev. E 61, 5743 (2000).

9. N. Horiuchi et al., Phys. Rev. E 74, 056601 (2006).

10. T. Ochiai and K. Ohtaka, Opt. Express 16, 7397 (2006).

11. D. Yu. Sergeeva, A. A. Tishchenko, and M. N. Strikhanov, Nucl. Instrum. Methods Phys. Res., Sect. B 402, 206 (2017).

12. L.-B. Kong et al., Sci. Rep. 5, 8772 (2015).

13. Z. Ping et al., Chin. Phys. B 21, 104102 (2012).

14. A. A. Tishchenko and D. Yu. Sergeeva, Phys. Rev. B 100, 235421 (2019).

15. M. I. Ryazanov, M. N. Strikhanov, and A. A. Tishchenko, Nucl. Instrum. Methods Phys. Res., Sect. B 266, 3811 (2008).

Translated by G. Dedkov 